\title{
Action de la Réserpine sur le parasitisme du Rat par Strongyloides ratti.
}

\section{Approche du mécanisme de l'hypocorticostéronémie vermineuse}

\author{
par J. BAILENGER et G. FARAGGI \\ Laboratoire de Zoologie et Biologie animale, Faculté de Pharmacie, \\ place de la Victoire, F 33000 Bordeaux
}

\section{Résumé.}

La Réserpine, injectée par voie sous-cutanée à la dose moyenne de $0,800 \mathrm{mg} / \mathrm{kg} / \mathrm{jour}$, à des rats expérimentalement infestés par Strongyloides ratti maintient leur parasitisme à un niveau très élevé et empêche la self-cure qui marque l'évolution normale de cette parasitose.

Cet alcaloïde s'oppose donc aux mécanismes qui aboutissent à l'expulsion des vers.

A l'opposé de l'hypocorticostéronémie qui caractérise l'état des rats porteurs de Strongyloides ratti, la Réserpine provoque chez ceux-ci une hypercorticostéronémie.

L'hypercorticostéronémie produite en réponse aux injections de Réserpine est moins importante chez les rongeurs porteurs de Strongles que chez ceux qui en sont indemnes. Cette différence s'observe aussi bien lorsque le traitement est poursuivi 10 jours, que lorsqu'il est limité à deux injections localisées à la phase d'hypocorticostéronémie parasitaire.

Cette action hypercorticostéronémiante s'apparenterait à un mécanisme neuromodérateur par lequel la Réserpine déprimerait les voies inhibitrices qui contrôlent la sécrétion du Cortico-releasing-factor par l'hypothalamus. Le freinage thérapeutique des facteurs inhibiteurs s'opposerait ainsi à leur stimulation déclenchée par le parasite d'où la différence entre les intensités réactionnelles des rats normaux et des rats parasités. Cette hypothèse rejoint celle à laquelle conduit l'exploration fonctionnelle de l'axe hypothalamo-hypophysocorticosurrénal. 
Cette expérience conduit donc à deux interprétations quant au mécanisme de la self-cure parasitaire :

- les strongles provoquent une hypocorticostéronémie par inhibition de la sécrétion hypothalamique du Cortico-releasing-factor en intervenant sur les mécanismes nerveux de sa régulation;

-- l'hypocorticostéronémie va de pair avec la self-cure; l'empêcher revient à s'opposer au déparasitage spontané. Elle apparaît comme un élément essentiel dans le mécanisme de celui-ci.

\section{Summary.}

Action of Reserpine on parasitism of the Rat by Strongyloides ratti : Attempt to explain the mechanism of verminous hypo-corticosteronemy.

Reserpine, administered to rats infested experimentally with Strongyloides ratti by sub-cutaneous injections at the average dose of $0.800 \mathrm{mg} / \mathrm{kg} /$ per day, maintains their parasitism at a very high level and prevents the self-cure which marks the normal evolution of this parasitosis.

This alkaloid therefore goes against the mechanism which leads to the expulsion of the worms.

Unlike the hypocorticosteronemy which characterizes the state of rats carrying Strongyloides ratti, Reserpine causes a hypercorticosteronemy.

The hypercorticosteronemy produced in response to the injections of Reserpine is less important in the rodents carrying Strongylus than in those which do not have it. This difference is also found both when the treatment is continued for 10 days and when it is limited to two localised injections at the phase of parasitical hypocorticosteronemy.

This hypercorticosteronemying action would seem to be connected with a neuromoderating mechanism through which Reserpine depresses the inhibiting channels which control the secretion of the cortico-releasing-factor through the hypothalamus. The therapeutical slowing down of the inhibiting factors would thus seem to oppose their stimulation started by the parasite, whence the difference between the reactional intensities of normal rats and rats with parasites. This hypothesis rejoins that arrived at through the functional exploration of the hypothalamo-hypophyso-corticosurrenal axis.

This experiment therefore gives rise to two interpretations with regard to the mechanism of the parasitical self-cure :

- the strongylus causes a hypocorticosteronemy through inhibition of the hypothalamic secretion of the Cortico-releasing-factor by acting on the nervous mechanism of its regulation;

- the hypocorticosteronemy is on a par with the self-cure; preventing it means going against the spontaneous deparasiting. It appears to be an essential element in the mechanism of this. 
L'hypocorticostéronémie qui se maintient pendant toute la durée de l'évolution digestive de Strongyloides ratti chez le Rat (Bailenger et Carcénac, 1974) nous a conduit à faire une exploration physiologique de l'axe hypothalamo-hypophyso-corticosurrénal des rats hébergeant ce Nématode (Bailenger et Faraggi, 1975). Cette exploration montre l'intégrité fonctionnelle de ce complexe endocrine et conduit à admettre une inhibition du mécanisme nerveux de la sécrétion du facteur hypophysotrope par l'hypothalamus.

On peut vérifier la valeur de cette notion en administrant aux animaux parasités une substance capable de déprimer les voies inhibitrices hypothalamiques qui contrôlent la sécrétion du facteur hypophysotrope. Une telle propriété a été reconnue à la Réserpine par Maickel, Westermann et Brodie (1961), Montanari et Stockham (1962), ainsi que par Munson (1963).

De leur côté, Mauss (1963), Rothwell, Dineen et Love (1971) notent que la Réserpine intensifie l'infestation parasitaire. Sharp et Jarret (1968) montrent qu'elle inhibe la self-cure des rats parasités par Nippostrongylus brasiliensis.

Il nous semble donc intéressant d'analyser les répercussions d'un traitement par la Réserpine sur le parasitisme et la corticostéronémie des rats expérimentalement infestés par Strongyloides ratti.

\section{Matériel et méthodes}

Rats blancs, mâles, souche Wistar, pathogen-free, $250 \mathrm{~g}$.

Parasite: Strongyloides ratti ; Nématode à pénétration cutanée ; dont les adultes ont une localisation digestive après migration tissulaire des larves ; il donne lieu à un déparasitage spontané en trois semaines.

Répartition des animaux par lots soumis à des traitements différents et tels que certains servent de témoins. Les lots sont eux-mêmes subdivisés pour éviter la répétition des prélèvements sanguins importants sur un même animal et les stress correspondants.

Pour éviter les interférences d'agressions dues à l'environnement, tous les rats sont maintenus isolés et placés dans des conditions rigoureusement identiques.

Infestation par balnéation dans les conditions précédemment indiquées (Bailenger et Carcénac, 1974).

Contrôle du parasitisme, prélèvements sanguins et dosage de la corticostéronémie ont été décrits (Bailenger et Carcénac, 1974). Le sang est régulièrement prélevé à 9 heures pour tenir compte du cycle nycthéméral du complexe hypothalamus-pituitaire-Cortex surrénal.

La Réserpine est dissoute dans une solution aqueuse stérile à $5 \%$ d'acide ascorbique de sorte que sa concentration finale soit de $3,33 \mathrm{mg}$ pour $100 \mathrm{ml}$. Au moment de l'emploi on la dilue au cinquième avec de l'eau salée à $9 \%$ stérile. Un léger floculat apparaît progressivement; il est très facile de le disperser par agitation. Cette solution est injectée par voie sous-cutanée. 
Le travail que nous présentons comporte trois expériences:

- un essai préliminaire pour essayer de préciser la dose et les modalités d'administration de cet alcaloïde, susceptibles d'avoir un retentissement sur le parasite sans trop altérer l'état général de l'hôte ;

- une expérience comportant un traitement continu d'une dizaine de jours ;

- une épreuve limitée à la période d'hypocorticostéronémie parasitaire.

\section{Résultats}

Expérience $\mathbf{n}^{\circ} \mathbf{1}$ (tableau I, graphiques $1,2,3$ ).

Les rats infestés par $15 i 000$ larves sont répartis en 4 lots de 3 animaux chacun. Les traitements sont diversifiés selon les lots:

$-0,500 \mathrm{mg} / \mathrm{kg}$; jours : $10-11-12 / 14-15 / 17-18-19$;

$-1,250 \mathrm{mg} / \mathrm{kg}$; jours : $10-11$; $0,800 \mathrm{mg} / \mathrm{kg}$; jours : $\quad 12 / 14-15 / 17-18-19$;

$-1,250 \mathrm{mg} / \mathrm{kg}$; jours : $10-11$;

- Acide ascorbique ; jours : 10-11 - $12 / 14-15 / 17-18-19$.

Cette expérience permet cinq constatations :

$1^{\circ}$ Maintien du parasitisme à un niveau très élevé. L'élévation du nombre d'œufs et de larves contenus dans les selles n'est pas immédiat; il n'est significatif qu'une vingtaines d'heures après la $2^{\circ}$ injection.

L'influence sur le parasitisme est en rapport avec la dose. C'est ainsi qu'au

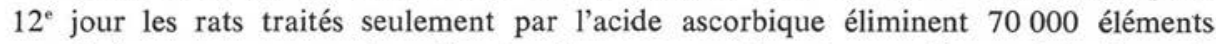
parasitaires par gramme de selles, tandis que pour les rats soumis aux injections de Réserpine on numère 108000 et 576000 parasites pour les doses respectives de 0,500 et $1,250 \mathrm{mg} / \mathrm{kg}$.

$2^{\circ} \mathrm{La}$ dose de $0,500 \mathrm{mg} / \mathrm{kg}$ ne semble pas être suffisante pour empêcher le déparasitage; ce résultat est atteint avec $0,800 \mathrm{mg} / \mathrm{kg}$.

$3^{\circ}$ La suppression d'un traitement est suivie d'une diminution brusque de l'excrétion des formes parasitaires de dissémination fécale. Elles passent de 126000 à 8000 (rats à $0,500 \mathrm{mg} / \mathrm{kg}$ ) et de 557000 à 127000 (rats à $0,800 \mathrm{mg} / \mathrm{kg}$ ).

$4^{\circ} \mathrm{La}$ première injection d'acide ascorbique provoque, dans les 24 heures, une stabilisation fugace du parasitisme en voie de régression. Cette réaction semble correspondre à un stress. La poursuite des injections ne modifie pas l'évolution normale du parasitisme. 
$5^{\circ}$ L'état général des animaux est affecté par le traitement ainsi qu'en témoigne l'évolution pondérale. Les témoins passent de 230 à $273 \mathrm{~g}$; pendant le même temps, les animaux traités par $0,500 \mathrm{mg} / \mathrm{kg}$ ne changent pas de poids; ceux qui reçoivent $1,250 \mathrm{mg} / \mathrm{kg}$ perdent $20 \mathrm{~g}$ en 2 jours; la poursuite de leur traitement par $0,800 \mathrm{mg} / \mathrm{kg}$ ralentit le dépérissement qui se poursuit toutefois et les amène à $173 \mathrm{~g}$ en fin d'expérience.

Ainsi, la dose de $0,500 \mathrm{mg} / \mathrm{kg}$ est relativement bien tolérée mais a une influence limitée sur le parasitisme ; $0,800 \mathrm{mg} / \mathrm{kg}$ est aux confins de la tolérance mais à une action manifeste sur l'évolution parasitaire. Les essais ultérieurs devront jouer sur ces deux doses.

TABLeau I. - Epreuve à la réserpine $\left(\mathrm{n}^{\circ} 1\right)$

\begin{tabular}{|c|c|c|c|c|}
\hline \multirow{3}{*}{$\begin{array}{l}\text { Temps } \\
\text { (jours) }\end{array}$} & \multirow{3}{*}{ Vit. C } & \multicolumn{3}{|c|}{$\begin{array}{l}\text { Evolution du parasitisme } \\
\text { (œufs }+ \text { larves } / g \text {. de selles) }\end{array}$} \\
\hline & & \multicolumn{3}{|c|}{ Réserpine $(\mathrm{mg} / \mathrm{kg})$} \\
\hline & & 0,500 & $\begin{array}{l}1,250 \\
0,800\end{array}$ & 1,250 \\
\hline $5 \ldots \ldots \ldots \ldots \ldots \ldots$ & 32800 & 33800 & 42800 & 28200 \\
\hline $7 \ldots \ldots \ldots \ldots \ldots \ldots$ & 212800 & 199000 & 199000 & 160200 \\
\hline $8 \ldots \ldots \ldots \ldots \ldots$ & 150000 & 181000 & 132500 & 150000 \\
\hline $10 \quad \ldots \ldots \ldots \ldots \ldots \ldots$ & 86000 & 72400 & 68000 & 81000 \\
\hline 11 (16 $\mathrm{h}$ après inject.) .. & 101700 & 51200 & 64500 & 114900 \\
\hline 12 (20 $\mathrm{h}$ après inject.) .. & 70000 & 108000 & 576000 & 746000 \\
\hline $14 \quad \ldots \ldots \ldots \ldots \ldots \ldots$ & 34800 & 86400 & 162000 & 93200 \\
\hline $15 \quad \ldots \ldots \ldots \ldots \ldots \ldots$ & 42000 & 126000 & 557000 & 33600 \\
\hline $17 \quad \ldots \ldots \ldots \ldots \ldots \ldots$ & $15000(\mathrm{~b})$ & 8000 & 127000 & 6800 \\
\hline $18 \ldots \ldots \ldots \ldots \ldots \ldots$ & 3600 & 2000 & 23000 & 4500 \\
\hline $19 \quad \ldots \ldots \ldots \ldots \ldots \ldots$ & 5000 & 2000 & 48000 & 2000 \\
\hline $20 \ldots \ldots \ldots \ldots \ldots \ldots$ & 3400 & - & 340000 & - \\
\hline
\end{tabular}

a) Début du traitement.

b) Injection de $0,8 \mathrm{mg} / \mathrm{kg}$ de réserpine.

\section{Doses}

$0,500 \mathrm{mg} / \mathrm{kg}$

( $1,250 \mathrm{mg} / \mathrm{kg}$

$0,800 \mathrm{mg} / \mathrm{kg}$

$1,250 \mathrm{mg} / \mathrm{kg}$
Jours

$10-11-12 * / 14-15 * * / 17-18-19$

$10-11$

10-11

* Interruption de 40 heures. $* *$ Interruption de 55 heures. 
Expérience $\mathbf{n}^{\circ} 2$ (tableau II, graphique 4).

Soixante-dix rats mâles de $250 \mathrm{~g}$ sont répartis en 6 lots. Trois d'entre eux servent de témoins, les autres sont infestés par 12000 larves. Dans chaque cas un lot n'est pas traité, l'autre reçoit la Réserpine à des doses alternées de 0,500 et $0,800 \mathrm{mg} / \mathrm{kg}$ :

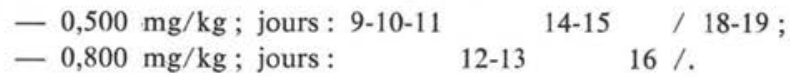

TABLEAU II. - Epreuve à la réserpine $\left(\mathrm{n}^{\circ} 2\right)$

\begin{tabular}{|c|c|c|c|c|c|c|c|c|}
\hline \multirow{3}{*}{$\begin{array}{l}\text { Temps } \\
\text { (jours) }\end{array}$} & \multicolumn{3}{|c|}{$\begin{array}{l}\text { Evolution du parasitisme } \\
\text { (œufs + larves/g. de selles) }\end{array}$} & \multicolumn{5}{|c|}{$\begin{array}{l}\text { Corticostéronémie } \\
(\mathrm{ug} / 100 \mathrm{ml})\end{array}$} \\
\hline & \multirow{2}{*}{$\begin{array}{l}\text { Non } \\
\text { traités }\end{array}$} & \multirow{2}{*}{ Vit. C } & \multirow{2}{*}{ Réserp. } & \multicolumn{2}{|c|}{ Non infestés } & \multicolumn{3}{|c|}{ Infestés } \\
\hline & & & & $\begin{array}{l}\text { Non } \\
\text { traités }\end{array}$ & Réserp. & $\begin{array}{c}\text { Non } \\
\text { traités }\end{array}$ & Vit. C & Réserp. \\
\hline 5 & 16400 & 24000 & 14000 & - & - & - & - & - \\
\hline $7 \ldots \ldots$ & 259000 & 300000 & 199000 & - & - & - & - & - \\
\hline $9 \ldots \ldots$ & 154000 & 170500 & 197500 & - & - & 13,6 & 13,6 & - \\
\hline $11 \ldots \ldots$ & 132000 & 139500 & 122000 & - & - & 11,7 & 11,2 & $-(a)$ \\
\hline $12 \ldots \ldots$ & - & - & - & 14,5 & - & - & - & - \\
\hline $13 \ldots \ldots$ & 65500 & 75500 & 447500 & - & - & 6,2 & 10 & - \\
\hline $14 \ldots \ldots$ & - & - & - & - & 37 & - & - & 20,2 \\
\hline $15 \ldots \ldots$ & 37000 & 43200 & 292000 & - & - & - & - & - \\
\hline $18 \ldots \ldots$ & 2000 & 6000 & 362000 & & - & 6,5 & 7 & - \\
\hline $19 \ldots \ldots$ & - & - & - & - & - & - & - & - \\
\hline $20 \ldots \ldots$ & 0 & 0 & 262000 & 15 & - & - & - & - \\
\hline
\end{tabular}

a) Début de traitement.

- Calendrier des traitements: doses alternées de 0,500 et $0,800 \mathrm{mg} / \mathrm{kg}$.

$0,500 \mathrm{mg} / \mathrm{kg}$ : 9-10-11 14-15/18-19 jours.

$0,800 \mathrm{mg} / \mathrm{kg}$ : $12-13 \quad 16 /{ }^{\text {* }}$ jours.

- Interruption de 40 heures.

$\mathrm{Au}$ troisième lot on injecte la solution ascorbique qui sert de véhicule à la Réserpine.

On note :

- Maintien du parasitisme: les déjections des animaux traités par la Réserpine renferment encore, par gramme, 262000 éléments parasitaires alors que les témoins non traités ou seulement soumis aux injections d'acide ascorbique sont déparasités.

- Hypercorticostéronémie provoquée par la Réserpine chez les animaux infestés ou non. Mais la réponse est plus importante chez ces derniers: la corticostéronémie est de $37 \mu \mathrm{g}$ (animaux témoins) contre 20,2 $\mu \mathrm{g}$ (rats parasités). L'acide ascorbique qui sert de solvant à la Réserpine n'a que peu ou pas d'influence: la 


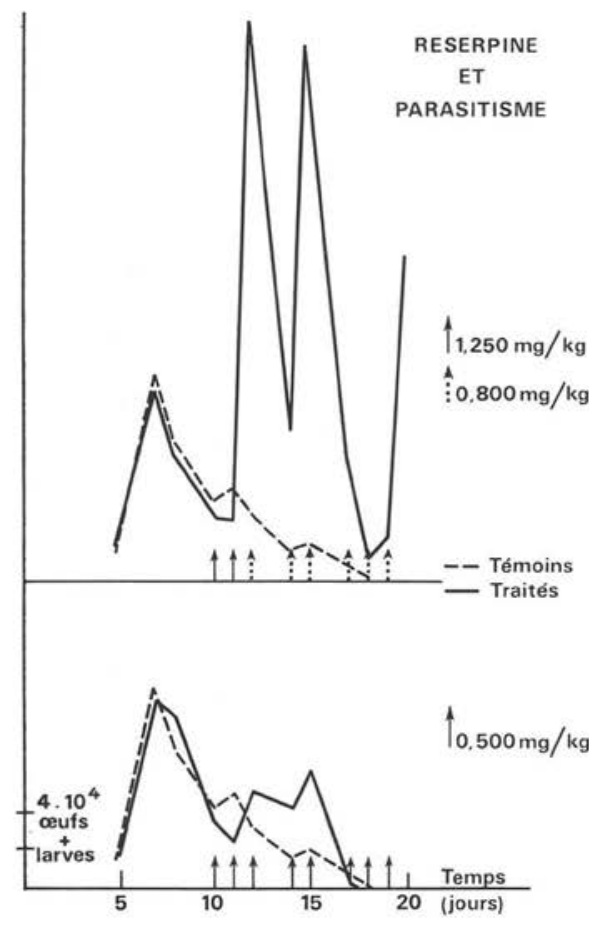

Graphiques 1 et 2

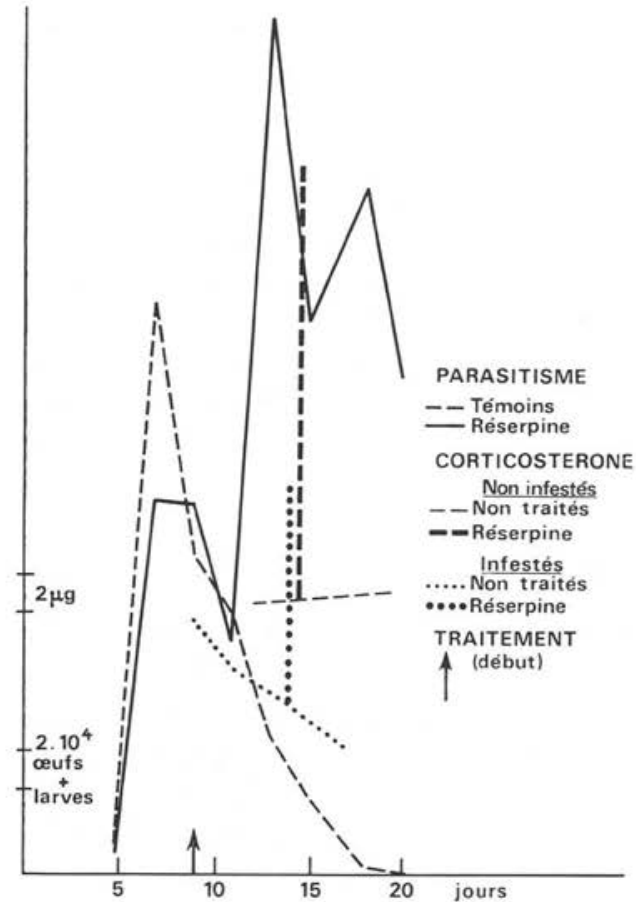

Réserpine et parasitisme

(Expérience $\mathrm{n}^{\circ} 1$ )

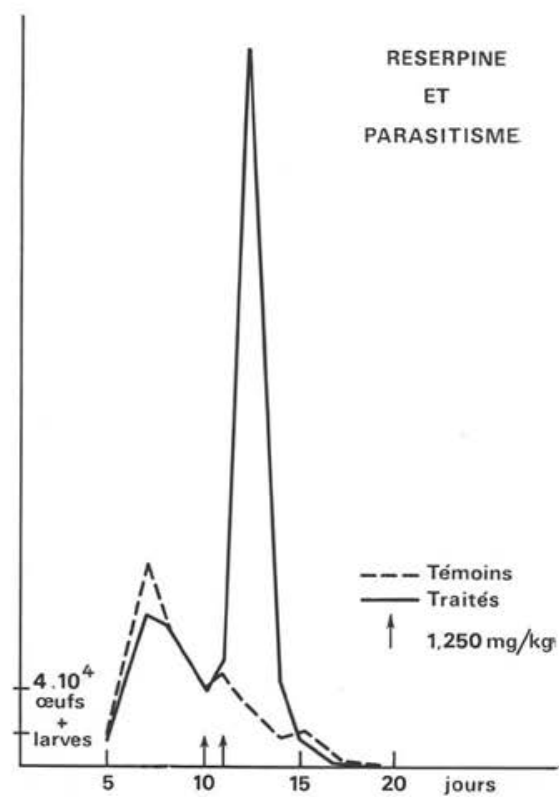

Graphique 3

Réserpine, parasitisme et corticostéronémie (Expérience $\mathrm{n}^{\circ} 2$ ) 
corticostérone est de 6,2 $\mu \mathrm{g}$ (animaux infestés non traités) et $10 \mu \mathrm{g}$ (animaux infestés traités par la vitamine C).

Expérience $\mathbf{n}^{\circ} 3$ (tableau III).

Le traitement par la Réserpine est limité à la période d'hypocorticostéronémie parasitaire et ne comporte que 2 injections.

TABLEAU III. - Epreuve à la réserpine (n’ 3 )

\begin{tabular}{|c|c|c|c|c|c|c|c|}
\hline & \multirow{3}{*}{$\begin{array}{l}\text { Temps } \\
\text { (jours) }\end{array}$} & \multicolumn{2}{|c|}{$\begin{array}{l}\text { Evolution du parasitisme } \\
\text { (œufs }+ \text { larves } / g \text { de selles) }\end{array}$} & \multicolumn{4}{|c|}{$\begin{array}{l}\text { Corticostéronémie } \\
(\mathrm{ug} / 100 \mathrm{ml})\end{array}$} \\
\hline & & \multirow{2}{*}{ Non traités } & \multirow{2}{*}{ Réserpine } & \multicolumn{2}{|c|}{ Non infestés } & \multicolumn{2}{|c|}{ Infestés } \\
\hline & & & & $\begin{array}{l}\text { Non } \\
\text { traités }\end{array}$ & Réserp. & $\begin{array}{l}\text { Non } \\
\text { traités }\end{array}$ & Réserp. \\
\hline 5 & $\ldots \ldots \ldots \ldots$ & 16400 & 28300 & - & - & - & - \\
\hline 7 & $\ldots \ldots \ldots \ldots$ & 259000 & 230000 & - & - & - & - \\
\hline 9 & $\ldots \ldots \ldots \ldots$ & 154000 & 209000 & - & - & 13,6 & - \\
\hline 11 & $\ldots \ldots \ldots \ldots$ & 132000 & 130000 & - & - & 14,7 & - \\
\hline 12 & $\ldots \ldots \ldots$ & - & - & 14,5 & - & - & - \\
\hline 13 & (a) $\ldots \ldots$ & 65500 & 76000 & - & - & 6,2 & - \\
\hline 15 & $\ldots \ldots \ldots$ & 37600 & 66000 & - & $46(b)$ & - & $32(b)$ \\
\hline 18 & $\ldots \ldots \ldots$ & 2000 & 8500 & - & - & 6,5 & - \\
\hline 20 . & $\ldots \ldots \ldots$ & 0 & - & 15 & 一 & - & - \\
\hline
\end{tabular}

a) $0,500 \mathrm{mg} / \mathrm{kg}$ (13* jour); $0,800 \mathrm{mg} / \mathrm{kg}$ (14 $4^{*}$ jour $)$.

b) Dosage effectué 16 heures après la $2^{e}$ injection.

40 rats sont répartis en 4 lots. Deux non infestés sont témoins: 2 infestés par 12000 larves: l'un non traité permet de suivre l'évolution de la corticostéronémie ; l'autre est réservé pour l'épreuve à la Réserpine qui consiste en une première injection de $0,500 \mathrm{mg} / \mathrm{kg}$ et une seconde, le lendemain, de $0,800 \mathrm{mg} / \mathrm{kg}$.

Cette épreuve est accomplie aux $13^{\circ}-14^{\mathrm{e}}$ jours alors que la corticostéronémie des rats porteurs de Strongles atteint $6,2 \mu \mathrm{g}$ contre $14,5 \mu \mathrm{g}$ en l'absence d'infestation.

Seize heures après la deuxième injection de Réserpine, la corticostéronémie passe de 6,2 à $32 \mu \mathrm{g}$ (rats parasités) et de 14,5 à $46 \mu \mathrm{g}$ (témoins).

\section{Discussion}

Cette expérience nous a permis de dégager un certain nombre de notions qu'il convient d'interpréter :

$1^{\circ}$ Un traitement prolongé par la Réserpine maintient le parasitisme des rats par Strongyloides ratti et, à dose suffisante, est capable d'empêcher la guérison spontanée.

Ces résultats confirment ceux obtenus par Mauss (1963) en travaillant sur la trichinose de la Souris, par Sharp et Jarrett (1968) avec des rats parasités par Nip- 
postrongylus brasiliensis et par Rothwell (1971) au cours d'expériences sur l'évolution de Trichostrongylus colubriformis chez le Cobaye immunisé.

La Réserpine s'oppose donc aux mécanismes qui aboutissent à l'expulsion de ces vers.

$2^{\circ}$ La Réserpine déclenche une hypercorticostéronémie importante : il suffit de deux injections espacées de 24 heures, cette hypersécrétion hormonale se maintient après des injections répétées.

Nous retrouvons la relation hypercorticostéronémie/maintien du parasitisme, relation sur laquelle nous avons déjà attiré l'attention (Bailenger et Faraggi, 1975). Nous apportons ainsi une confirmation à notre hypothèse: l'hypocorticostéronémie, réaction aspécifique déclenchée par le parasite, conditionne les défenses aspécifiques et spécifiques qui aboutissent à l'expulsion de celui-ci.

Pour expliquer l'inhibition de la self-cure des rats parasités par Nippostrongylus brasiliensis et traités par la Réserpine, Sharp et Jarrett (1968) admettent que l'action de cet alcaloïde résulterait de sa propriété d'inhiber la fixation tissulaire des amines biogènes, principes auxquels certains auteurs font jouer un rôle important dans l'élimination des vers bien qu'il n'existe pas de preuves formelles de leur intervention.

Il n'y a pas opposition entre l'hypothèse que nous avons formulée et celle qui fait entrer en jeu les amines biogènes, car elles se situent à des niveaux différents ; l'une pouvant être la conséquence de l'autre: si l'intervention des amines biogènes se confirmait, on pourrait concevoir que l'hypercorticostéronémie agisse en empêchant la libération des amines biogènes protectrices, libération qui est au contraire accrûe par l'hypocorticostéronémie. Ceci, indépendamment de l'action pharmacologique spécifique de la Réserpine sur la déplétion des amines biogènes.

$3^{\circ}$ L'hypercorticostéronémie provoquée par l'injection de Réserpine est moindre chez les rats parasités que chez les rats normaux.

Cette observation nous ramène au mécanisme de l'hypocorticostéronémie d'origine parasitaire.

En premier lieu, il convient de rappeler que tous les auteurs sont d'accord pour admettre que l'action de la Réserpine sur le cortex-surrénal s'exerce par l'intermédiaire de l'axe hypothalamo-pituitaire: quel que soit le sens de cette action, elle ne se produit pas après hypophysectomie.

La notion d'une hypersécrétion cortico-surrénale à laquelle conduisent nos travaux, est également signalée chez le Rat mâle par Montanari et Stockham (1962) et ceci en dehors de toute intervention parasitaire. Ces auteurs notent que ce résultat n'est obtenu qu'avec des doses sédatives et que, parmi les alcaloïdes du Rawolfia, seuls ceux qui donnent une sédation augmentent l'activité du cortex surrénal. Pour eux, ainsi que pour Maickell et coll. (1961) de même que pour Munson (1963), l'hypercorticostéronémie s'apparente à un mécanisme neuro-modérateur par lequel la Réserpine déprimerait les voies inhibitrices qui contrôlent la sécrétion du facteur hypophysotrope par l'hypothalamus.

Revenons maintenant sur le fait d'une différence entre les intensités réactionnelles des rats normaux et des rats parasités: l'hypercorticostéronémie de ceux-ci est 
significativement moindre que celle des animaux témoins. Un freinage qui gêne l'action de la Réserpine semble donc caractériser l'état parasitaire.

Ces résultats nous ramènent à l'hypothèse que nous ont amené à poser les tests d'exploration fonctionnelle de l'axe hypothalamo-hypophyso-corticosurrénal (Bailenger et Faraggi, 1975) : les Strongles, lors de leur localisation digestive provoquent une inhibition de la sécrétion hypothalamique. Cette inhibition ne peut être que partiellement levée par la Réserpine d'où une hypercorticostéronémie moindre chez les rats malades que chez les rats sains.

Ainsi, le traitement par la Réserpine apporte des arguments supplémentaires appuyant notre hypothèse d'après laquelle le parasite déprime la sécrétion par l'hypothalamus du cortico-releasing-factor en intervenant sur les mécanismes nerveux de sa régulation.

De cette expérience il convient aussi de retenir que l'hypocorticostéronémie va de pair avec la self-cure; l'empêcher revient à s'opposer au déparasitage spontané ; elle apparaît comme un élément essentiel dans le mécanisme de celui-ci.

\section{REMERCIEMENTS}

La souche de Strongyloides ratti est entretenue à l'Unité de Recherche sur l'Immunologie des infections parasitaires (I.N.S.E.R.M.), 33000 Bordeaux Nous remercions MM. les $\mathrm{P}^{\mathrm{rs}}$ Pautrizel et Tribouley d'avoir bien voulu la mettre à notre disposition.

Nous remercions les Laboratoires C.I.B.A. qui nous ont aimablement adressé le produit pur ayant servi à cette expérimentation.

Cette expérimentation a pu être réalisée grâce à une subvention de la Fondation pour la Recherche médicale française.

\section{Bibliographie}

Batlenger (J.) et CARCenac (F.), 1974. - Répercussion du parasitisme par Strongyloides ratti sur la sécrétion des gluco-corticostéroides chez le Rat. Int. J. Parasit., 4, 307-310.

Bailenger (J.) et Faraggi (G.), 1975. - Mécanisme de l'action hypocorticostéronémiante de Strongyloides ratti chez le Rat. Ann. Parasit. hum. comp., 50, 187-197.

Maickel (R.P.), Westermann (E. O.) et Brodie (B. B.), 1961. - Effects of Reserpine and clod-exposure on pituitary adrenocortical function in rats. J. Pharm. exp. Ther., 134, 167-175.

Montanari (R.) et Stockham (M. A.), 1962. - Effects of simple and repeated doses of Reserpine on the secretion of Adrenocorticotropic hormone. Brit. J. Pharmacol., $18,337-345$.

Munson (P. L.), 1963. - Pharmacology of neuroendocrine blocking agents. Advanc. neuroendocr., 427-444.

Mauss (E. A.), 1963. - Effect of Reserpine on Trichinella spiralis, infection in rats. Exp. Parasit., 13, 244-252.

Rothwell (T. L. W.), Dineen (J. K.) et Love (R. J.), 1971. - The role of Pharmacologically active amines in resistance to Trichostrongylus colubriformis in the guinea pig. Immunology, 21, 925.

Sharp (N. C.C.) et JARretT (W.F.H.), 1968. - Inhibition of immunological expulsion of helminths by reserpine. Nature, 218, 1161-1162. 